\title{
The Population Structure of Trypanosoma cruzi: Expanded Analysis of 54 Strains Using Eight Polymorphic CA-repeat Microsatellites
}

\section{Riva P Oliveira, Anna Izabel R Melo*, Andréa M Macedo*, Egler Chiari**, Sérgio DJ Pena*/+}

\author{
Departamento de Ciências Biológicas, Universidade Federal de Ouro Preto, Ouro Preto, MG, Brazil \\ *Departamento de Bioquímica e Imunologia **Departamento de Parasitologia, Universidade Federal de Minas \\ Gerais, Caixa Postal 486, 30161-970 Belo Horizonte, MG, Brazil
}

Recently we cloned and sequenced the first eight Trypanosoma cruzi polymorphic microsatellite loci and studied 31 clones and strains to obtain valuable information about the population structure of the parasite. We have now studied 23 further strains, increasing from 11 to 31 the number of strains obtained from patients with chronic Chagas disease. This expanded set of 54 strains and clones analyzed with the eight microsatellites markers confirmed the previously observed diploidy, clonal population organization and very high polymorphism of T. cruzi. Moreover, this new study disclosed two new features of the population genetic structure of $\mathrm{T}$. cruzi. The first was the discovery that, similarly to what we had previously shown for strains isolated from insect vectors, mammals and humans with acute disease, isolates from patients in the chronic phase of Chagas disease could also be multiclonal, albeit at a reduced proportion. Second, when we used parsimony to display the genetic relationship among the clonal lineages in an unrooted Wagner network we observed, like before, a good correlation of the tree topography with the classification in three clusters on the basis of single locus analysis of the ribosomal RNA genes. However, a significant new finding was that now the strains belonging to cluster 2 split in two distant sub-clusters. This observation suggests that the evolutionary history of T. cruzi may be more complex than we previously thought.

Key words: Trypanosoma cruzi - microsatellites - genetic typing - Chagas disease

Natural populations of Trypanosoma cruzi exhibit extensive genetic polymorphism (reviewed by Macedo \& Pena 1998). This variation has been characterized by the use of isoenzymes (Miles et al. 1978, Tibayrenc et al. 1986), DNA fingerprinting (Macedo et al. 1992), Random Amplified Polymorphic DNA (RAPD; Tibayrenc et al. 1993, Gomes et al. 1998), Simple Sequence Repeat-anchored PCR (SSR-PCR; Oliveira et al. 1997, Gomes et al. 1998), Low-stringency Single Specific Primer-PCR (LSSP-PCR; Vago et al. 1996) and molecular karyotyping (Henriksson et al. 1993). Also, single locus analysis of the ribosomal RNA genes (LSU-rDNA) and mini-exon genes (Souto \& Zingales 1993, Souto et al. 1996) has suggested the split of $T$. cruzi strains into three

\footnotetext{
This work was supported by Pronex (Finep), CNPq and Fapemig.

${ }^{+}$Corresponding author. Fax: +55-31-227.3792. E-mail: spena@dcc.ufmg.br

Received 9 June 1999

Accepted 9 August 1999
}

major clusters (1, 2 and 1/2) that possibly represent sub-species (Souto et al. 1997).

Recently, we cloned and sequenced the first eight T. cruzi polymorphic microsatellite loci and studied 31 clones and strains to obtain valuable information about the population structure of the parasite (Oliveira et al. 1998). We have now studied 23 further strains, increasing from 11 to 31 the number of strains obtained from patients with chronic Chagas disease. This expanded set of 54 strains and clones analyzed with the eight microsatellites markers disclosed interesting new features of the population genetic structure of $T$. cruzi.

\section{MATERIAL AND METHODS}

Parasites - The 54 strains and clones of T. cruzi studied were isolated from a variety of vectors or mammalian hosts and were kindly provided by Prof. Egler Chiari, from the Laboratório de Biologia do Trypanosoma cruzi, Departamento de Parasitologia, UFMG. Epimastigote forms were grown in LIT (liver infusion tryptose) medium. The parasites were collected and stored $-70^{\circ} \mathrm{C}$ until standard DNA extraction with phenol-chloroform was done as previously described (Macedo et al. 1992). 
PCR amplification and detection of microsatellite alleles - The microsatellite loci analyzed are shown in Table I. Primers sequences have been published previously (Oliveira et al. 1998). One primer of each pair was 5'-labeled with fluorescein. After PCR, the amplified microsatellites were loaded on a $6 \%$ denaturing polyacrylamide gel and analyzed on an ALF sequencer (Pharmacia) using the Allelinks software. To determinate the allele size the samples were directly compared with band sizes from an allelic ladder prepared by amplification of an artificial mixture of DNA from $60 \mathrm{~T}$. cruzi strains.

Construction of a phylogenetic tree - To make phylogenetic inferences, we assumed a stepwise mutation model for the microsatellites. As a measure of genetic distance between any two strains we used the minimum number of mutational steps necessary to transform one into the other. The microsatellite multilocus genotypes were transformed into 0 and 1 characters using the FACTOR program from the PHYLIP package version 3.57c (Felsenstein 1993). These data were then used to construct unrooted Wagner parsimony trees using the MIX program also from the PHYLIP package. The significance levels of the branching in the Wagner network was achieved by bootstrapping $(1,000$ reiterations) using the program SEQBOOT from the PHYLIP software package.

\section{RESULTS}

Polymorphism of CA-repeats in T. cruzi - We first performed an analysis of seven different clones with the eight CA-repeat microsatellite loci. All these clones showed amplification of only one or two fragments of different sizes in all loci (Fig. 1A). We interpreted this as further indication that T. cruzi is diploid; the two-peak patterns were interpreted as heterozygosity at the respective loci and the one-peak patterns indicated homozigosity. We next typed 47 different strains at all the eight microsatellite loci. Again we observed amplification of one or two fragments peaks for most strains. However, fifteen strains consistently showed amplification of three or four major fragments of different size in two or more loci (Fig. 1B), implying that they were multiclonal. In our previous study with microsatellites (Oliveira et al. 1998) we had only observed more than two peaks in strains obtained from insect vectors, wild mammals or human patients in the acute phase of Chagas disease. Now, with the expanded number of strains from chronic patients we could now observe several cases of more than two peaks, although still at a smaller proportion (Table II).

Tests for Hardy-Weinberg proportions and linkage disequilibrium - T. cruzi is believed to have a clonal population structure (Tibayrenc et al. 1986), with absence of sexual reproduction. We further
A

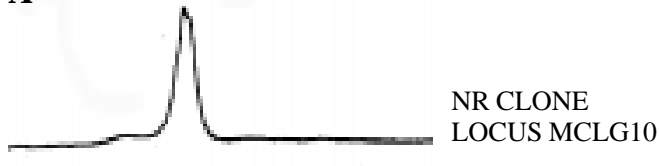

S03 CLONE LOCUS MCLG10
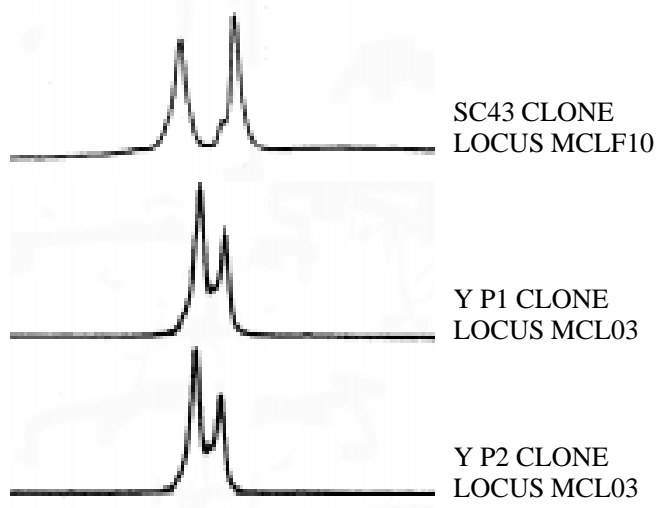

\section{B}
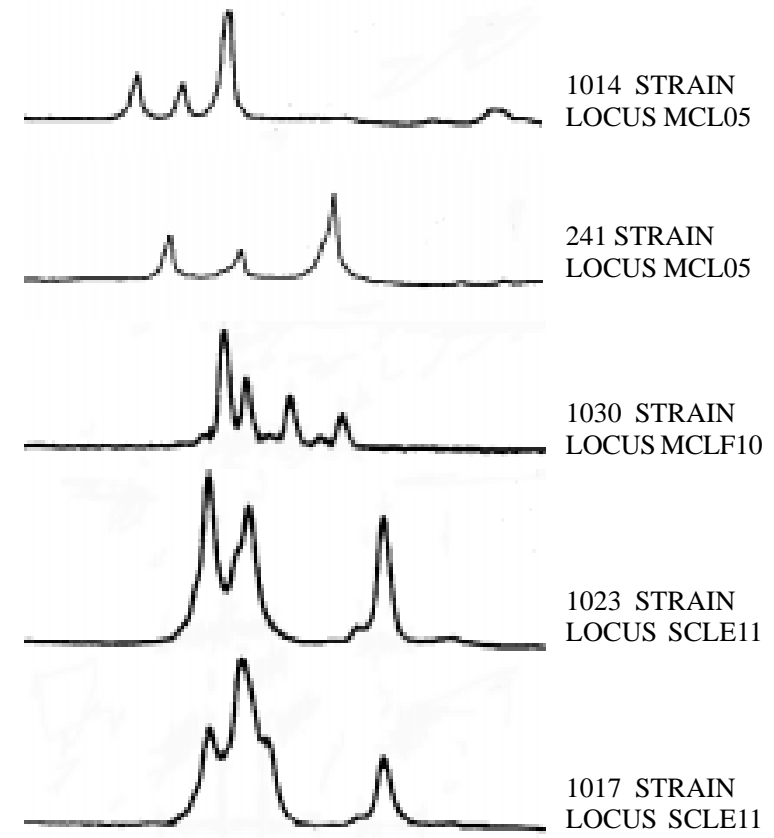

Fig. 1- A: electrofluorogram of microsatellite alleles of Trypanosoma cruzi clones showing the amplification of one or two peaks; B: electrofluorogram of microsatellite alleles of $T$. cruzi strains showing the amplification of three or four peaks. The microsatellite loci amplified are indicated in parenthesis. 
TABLE I

Microsatellites markers isolated from Ttypanosoma cruzi

\begin{tabular}{lcccc}
\hline Marker & $\begin{array}{c}\text { Size range } \\
\text { allele (bp) }\end{array}$ & $\begin{array}{c}\text { Number of } \\
\text { alleles found }\end{array}$ & $\begin{array}{c}\text { Observed } \\
\text { heterozygosity }\end{array}$ & $\begin{array}{c}\text { Expected } \\
\text { heterozygosity }\end{array}$ \\
\hline MCLE01 & $110-156$ & 16 & 0.66 & 0.86 \\
MCLE08 & $118-130$ & 7 & 0.31 & 0.78 \\
SCLE10 & $233-291$ & 14 & 0.82 & 0.86 \\
SCLE11 & $139-165$ & 11 & 0.64 & 0.84 \\
MCLF10 & $180-194$ & 6 & 0.49 & 0.61 \\
MCLG10 & $149-187$ & 9 & 0.28 & 0.50 \\
MCL03 & $257-319$ & 14 & 0.64 & 0.89 \\
MCL05 & $194-228$ & 15 & 0.51 & 0.91 \\
\hline
\end{tabular}

TABLE II

Proportion of Trypanosoma cruzi strains with more than two microsatellite peaks according to their origin

\begin{tabular}{|c|c|c|}
\hline Sample & $\begin{array}{c}\text { Total } \\
\text { number }\end{array}$ & $\begin{array}{l}\text { Strains with more } \\
\text { than two peaks }\end{array}$ \\
\hline $\begin{array}{l}\text { Strains isolated from } \\
\text { non-human sources }\end{array}$ & 9 & $5(55 \%)$ \\
\hline $\begin{array}{l}\text { Strains isolated from patients } \\
\text { with acute Chagas disease }\end{array}$ & s & $3(38 \%)$ \\
\hline $\begin{array}{l}\text { Strains isolated from patients } \\
\text { with chronic Chagas disease }\end{array}$ & 30 & $7(25 \%)$ \\
\hline Total & 47 & $15(32 \%)$ \\
\hline
\end{tabular}

tested this notion with the microsatellite analysis of this expanded set of strains. Phenotypes for the eight microsatellite repeats were established for 39 of the T. cruzi strains or clones (the strains with more than two bands were excluded from this analysis), and the corresponding multilocal genotypes were inferred. Microsatellite data showed drastic departures from Hardy-Weinberg expectation $(\mathrm{P}<0.001)$. These can be appreciated by the striking difference seen between observed and expected heterozygosity in seven of eight microsatellite loci shown in Table I. Statistical tests for all the 28 pairwise comparisons possible for all eight microsatellite loci showed that all of these loci are associated in very strong linkage disequilibrium $(\mathrm{P}<0.001)$. Together, these observations reinforce the notion that the population structure of $T$. cruzi is clonal and that sexual reproduction is rare or absent.

Phylogenetic relationships - The genetic diversity of $T$. cruzi disclosed with the polymorphic CArepeat microsatellites was remarkable. None of the previous methods of genetic analysis illustrated so well the degree of genetic individuality in the protozoon. Among the 54 different $T$. cruzi strains and clones no single multilocus genotype was seen more than once. To investigate the phylogenetic relationship among $T$. cruzi strains based on the microsatellite analysis, we assumed a stepwise mutation model according to which microsatellites evolve by the gain or loss of one repetitive unit. As genetic distance we used the minimum number of mutational steps necessary to transform one strain into the other. A total of 39 strains and clones were analyzed by these procedure (the strains that displayed more that two peaks were excluded). The overall dispersion pattern based on microsatellite genotypes shows that the $T$. cruzi strains are genetically very distant from each other (Fig. 2). In general, we did not observe any clustering among the clones and strains in relation to geographical origin or the clinical features of Chagas disease. This is especially significant because most strains were from the same geographic area. The only exception was a very homogeneous cluster observed among S03, NR and SC43 clones that showed a difference of a single mutational event. These clones have been previously characterized by isoenzyme analysis as clonet 39 (Tibayrenc et al. 1986).

In the Wagner network, there was clear segregation of strains based on rRNA typing (Fig. 2). Strains classified as belonging to cluster 1 were found separated from the strains belonging to clusters 2 and 1/2. However, in contrast with our previously observed homogeneity of group 2 , there was a now a split of cluster 2, with two strains (Colombiana and 1001) placed distantly from the others belonging to groups $1 / 2$ and 2 (Fig. 2).

\section{DISCUSSION}

In concordance with our previous study (Oliveira et al. 1998), the observation that clones yielded only one or two microsatellite bands at the different loci strongly suggests that the species is diploid in most, if not all, of its genome. Most of the previous information supporting the diploidy is derived from zymodeme studies and hybridization on restricted DNA or chromosomal bands 


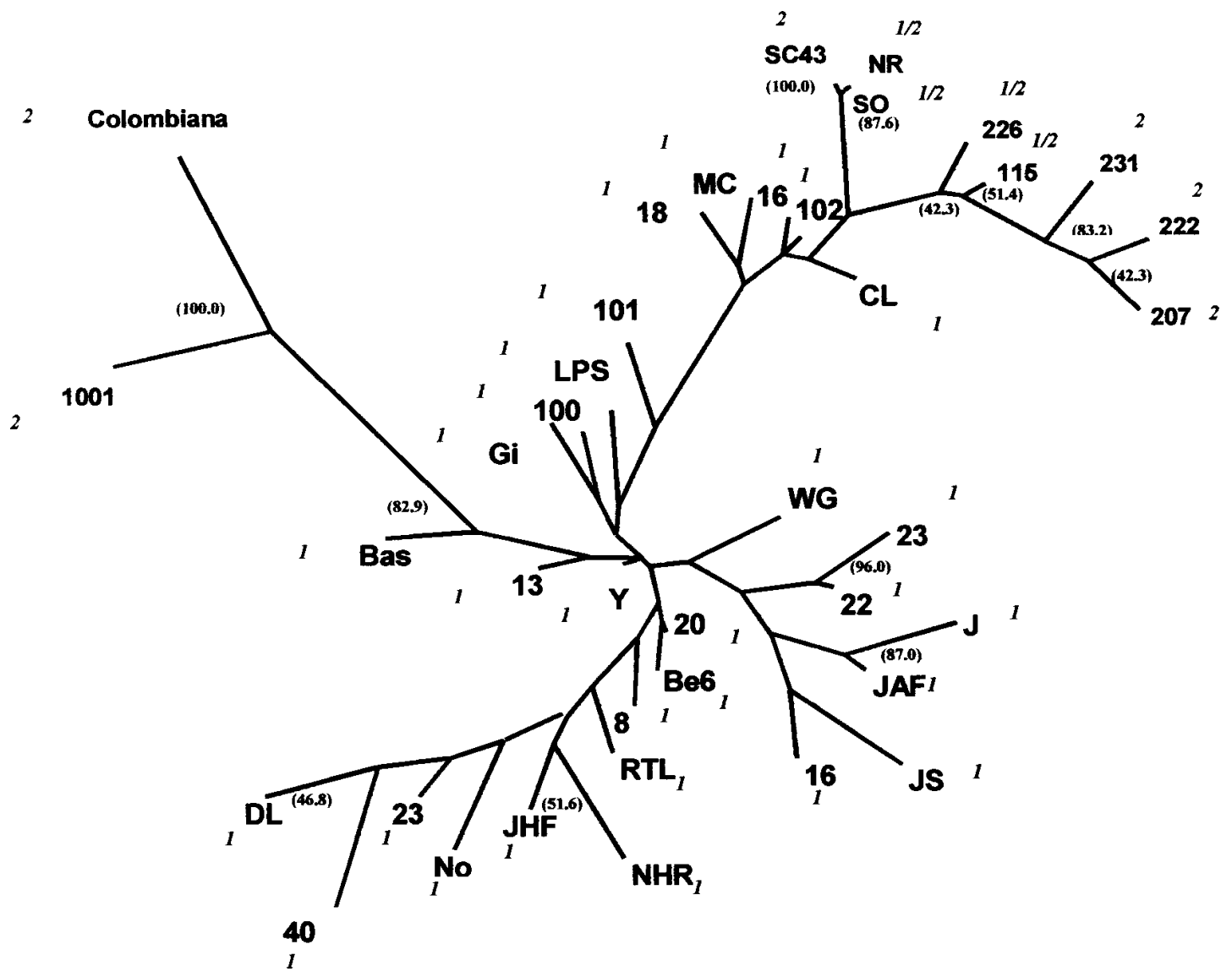

Fig. 2: unrooted Wagner network of 39 Trypanosoma cruzi strains and clones based on the amplification of eight polymorphic loci with (CA)n repeats. The classification of each strain indicated in italic $(1,2$ or 1/2) was based on the rRNA $24 \mathrm{~S} \alpha$ amplification. The figures in parenthesis indicate the number of times that the branch was observed in 100 bootstraps. Bootstrap values lower than 40 are not shown.

separated by PFGE (Tibayrenc et al. 1986, Gibson \& Miles 1986, Henriksson et al. 1993, 1995). Likewise, our results with this expanded set of strains agreed with our previous observations in showing drastic departures from Hardy-Weinberg expectations and strong linkage disequilibrium, suggesting that sexual reproduction is rare or absent in $T$. cruzi $i$ and that the population structure is clonal, as had been previously suggested on the basis of isoenzyme studies (Ayala 1993, Tibayrenc 1995, Tibayrenc et al. 1996). Thus, each clone represents a lineage that reproduces by binary division and remains stable for a large number of generations until a mutation occurs.

There were two important new findings in the present study. First, we observed a significant number of strains obtained from patients with chronic Chagas disease exhibiting more than two peaks in several microsatellite loci (7/31). In our previous work with microsatellites (Oliveira et al. 1998) we had seen more than two peaks in strains obtained from the insects, wild animals or acute patients (6/ 11) but not in those from chronic patients $(0 / 13)$. We interpret the presence of more than two peaks as evidence of strain multiclonality. Strains or isolates of $T$. cruzi are obtained by culturing parasites isolated from triatomid insect vectors or mammalian hosts, including humans. Patients in endemic areas may be infected by multiple contacts with different triatomids, and these triatomids, in turn, may feed on different infected individuals. This promiscuity propitiates the formation of multiclonal populations in hosts and vectors, leading to the isolation of corresponding multiclonal strains when grow in culture. We would expect that the prevalence of multiclonal strains would be much higher if we could avoid the necessity of growth in culture during strain isolation because this step most likely promotes loss of some clones incapable to adapt to the new in vitro environment. Anyway, 
the observation of multiclonal strains isolated from patients with chronic Chagas disease demonstrates that genetic heterogeneity of infection persists even after establishment of the disease, which is in perfect agreement with the predictions of our ClonalHistotropic hypothesis (Macedo \& Pena 1998).

Single locus analysis of the ribosomal RNA genes (LSU-rDNA) and mini-exon genes (Souto \& Zingales 1993, Souto et al. 1996) has suggested the split of $T$. cruzi strains into three major clusters (1, 2 and 1/2) that might represent sub-species (Souto et al. 1996). When in our previous microsatellite study (Oliveira et al. 1998) we used parsimony to display the genetic relationship among the clonal lineages in an unrooted Wagner network we observed a good correlation of clusters 1 and 2 with the tree topography. The four strains that belonged to cluster $2(222,231,1001$ and Colombiana) grouped together in one branch with high bootstrap values. It is interesting to observe that 1001 and Colombiana grouped together a little distant from the other strains. Moreover, the only two strains that belonged to $1 / 2$ (115 e 226) clustered with group 2 , suggesting that conceivably the latter might represent the ancestral state. In the present study we significantly increased the number of strains in the tree from 19 to 39 . Since we included mostly more strains from chronic patients, which are known to belong primarily to cluster 1 , only three of the 20 additional strains were from cluster 2 (increasing the total to seven) and one from cluster $1 / 2$ (increasing the total to three). The new expanded Wagner network still displays segregation of clusters 1 and 2, but there is the significant new finding that now the strains belonging to cluster 2 split in two distant sub-clusters, with strains 1001 and Colombiana separate from the rest in a branch with high bootstrap value. This observation suggests that the evolutionary history of $T$. cruzi may prove to be more complex than we previously thought. However, it still needs to be confirmed by the study of a larger number of strains belonging to cluster 2 .

In conclusion, genetic typing based on microsatellite polymorphisms typed by PCR amplification represent a powerful new tool for the understanding of the population structure and dynamics of $T$. cruzi. This approach has the advantage of being simple and fast. It can be applied to many different parasites permitting the discover of CA repeats and other microsatellites important for physical mapping in the $T$. cruzi genome project and other parasites included in the Parasite Genome Initiative supported by WHO (Zingales et al. 1997).

\section{ACKNOWLEDGMENTS}

To Afonso da Costa Viana for expert technical help.

\section{REFERENCES}

Ayala FJ 1993. Trypanosoma and Leishmania have clonal population structures of epidemiological significance. Biol Res 26: 47-63

Felsenstein J 1993. PHYLIP (Phylogeny Inference Package) version $3.5 \mathrm{c}$. Department of Genetics, University of Washington, Seattle.

Gibson WC, Miles MA 1986. The karyotype and ploidy of Trypanosoma cruzi. EMBO J 5: 1299-1305.

Gomes ML, Macedo AM, Pena SDJ, Chiari E 1998. Genetic relationship between Trypanosoma cruzi strains isolated from chronic chagasic patients on Southern Brazil as revealed by RAPD and SSR-PCR analysis. Acta Tropica 69: 99-109.

Henriksson J, Petterson U, Solari A 1993. Trypanosoma cruzi: correlation between karyotype variability and isoenzyme classification. Exp Parasitol 77: 334-348.

Henriksson J, Porcel B, Rydaker M, Ruiz A, Sabaj V, Galanti N, Cazzulo JJ, Frasch ACC, Petterson U 1995. Chromosome specific markers reveal conserved linkage groups in spite of extensive chromosomal size variation in Trypanosoma cruzi. $\mathrm{Mol}$ Biochem Parasitol 73: 63-74.

Macedo AM, Pena SDJ 1998. Genetic variability of Trypanosoma cruzi: implications for the pathogenesis of Chagas disease. Parasitol Today 14: 119-124.

Macedo AM, Martins MS, Chiari E, Pena, SDJ 1992. DNA Fingerprints of Trypanosoma cruzi: a new tool for characterization of strains and clones. $\mathrm{Mol}$ Biochem Parasitol 55: 147-154.

Miles MA, Souza A, Povoa M, Shaw JJ, Lainson R, Toye PJ 1978. Isozymic heterogeneity of Trypanosoma cruzi in the first autochthonous patients with Chagas disease in Amazonian Brazil. Nature 272: 819-821.

Morel C, Chiari E, Camargo EP, Mattei DM, Romanha AJ, Simpson L 1980. Strains and clones of Trypanosoma cruzi can be characterized by restriction endonucleases fingerprint of kinetoplast DNA molecules. Proc Natl Acad Sci USA 77: 6810-6814.

Oliveira RP, Broude NE, Macedo AM, Cantor CR, Smith CL, Pena SDJ 1998. Probing the genetic population structure of Trypanosoma cruzi with polymorphic microsatellites. Proc Natl Acad Sci USA 95: 3776-3780.

Oliveira RP, Macedo AM, Chiari E, Pena SDJ 1997. An alternative approach to evaluating the intraspecific genetic variability of parasites. Parasitol Today 13: 196-200.

Souto, RP, Zingales B 1993. Sensitive detection and strain classification of Trypanosoma cruzi by amplification of a ribosomal RNA sequence. $\mathrm{Mol}$ Biochem Parasitol 62: 45-52.

Souto RP, Fernandes O, Macedo AM, Campbell DA, Zingales B 1996. DNA markers define two major phylogenetic lineages of Trypanosoma cruzi. Mol Biochem Parasitol 83: 141-152.

Tibayrenc M 1995. Population genetics of parasitic protozoa and other microorganisms. Adv Parasitol 36: 47-115.

Tibayrenc M, Neubauer K, Barnabé C, Guerrini F, Skarecky D, Ayala F 1993. Genetic characterization 
of six parasitic protozoa: Parity between randomprimer DNA typing and multilocus enzyme electrophoresis. Proc Natl Acad Sci USA 90: 1335-1339.

Tibayrenc M, Ward Pl, Moya A, Ayala FJ 1986. Natural populations of Trypanosoma cruzi, the agent of Chagas disease, have a complex multiclonal structure. Proc Natl Acad Sci USA 83: 293-296.

Vago AR, Macedo AM Oliveira RP, Andrade LO, Chiari E, Galvão LMC, Reis D A, Pereira MES, Simpson AJG, Tostes S, Pena SDJ 1996. kDNA signatures of
Trypanosoma cruzi strains obtained directly from infected tissues. Am J Pathol 149: 2153-2159.

Weber JL, Wong C 1993. Mutation of human short tandem repeats. Hum Mol Genet 2: 1123-1128.

Zingales B, Rondinelli E, Degrave W, da Silveira JF, Levin M, Le Paslier D, Modabber F, Dobrokhotov B, Swindle J, Kelly JM, Aslund L, Hoheisel JD, Ruiz AM, Cazzulo JJ, Pettersson U, Frasch AC 1997. The Trypanosoma cruzi Genome Initiative. Parasitol Today 13: 16-22. 